\title{
"Agrobacterium yellow group" and Pseudomonas paucimobilis causing peritonitis in patients receiving continuous ambulatory peritoneal dialysis
}

\author{
RA SWANN, ${ }^{*}$ SJ FOULKES, $†$ B HOLMES,§ JB YOUNG, $\ddagger$ RG MITCHELL, ${ }^{*}$ ST REEDERS॥ \\ From the *Department of Bacteriology and Regional Public Health Laboratory, John Radcliffe Hospital, \\ Oxford, the †Department of Microbiology and $¥$ Renal Unit, Leeds General Infurmary, Leeds, the §National \\ Collection of Type Cultures, Central Public Health Laboratory, London, and the IRenal Unit, Churchill \\ Hospital, Oxford
}

SUMMARY Five cases of peritonitis occurred during continuous ambulatory peritoneal dialysis in which unusual yellow pigmented Gram negative bacteria were isolated in pure culture. Four of these isolates were identified as the so called "Agrobacterium yellow group." The remaining isolate was identified as Pseudomonas paucimobilis, an organism closely related to the Agrobacterium yellow group.

Peritonitis is the most important complication of continuous ambulatory peritoneal dialysis. Infection is usually due to skin organisms, most commonly Staphylococcus epidermidis. ${ }^{2}$ Many other bacteria and fungi have been documented as pathogens in this condition, including environmental organisms generally considered to have little or no pathogenicity.

The genus Agrobacterium contains several plant pathogens and has been speciated according to phytopathogenic effects. ${ }^{3}$ Several authors have expressed their disagreement with this classification. ${ }^{4}$ Two of these authors recognised three distinct species on the basis of numerical taxonomy of biochemical characteristics ( $A$ tumefaciens, $A$ rhizogenes, and $A$ rubi) and a fourth cluster of yellow pigmented isolates, which they tentatively designated the "Agrobacterium yellow group."' This taxon has not previously been reported as a cause of disease in man, and we report here four cases of peritonitis in patients receiving continuous ambulatory peritoneal dialysis caused by isolates apparently belonging to the Agrobacterium yellow group. A fifth patient had peritonitis caused by Pseudomonas paucimobilis, another yellow pigmented organism closely related to the Agrobacterium yellow group.

Accepted for publication 25 July 1985

\section{Case reports}

CASE 1

A 51 year old woman with chronic renal failure due to chronic pyelonephritis started continuous ambulatory peritoneal dialysis in April 1981 and was admitted with her first episode of peritonitis in July 1982. Blood and dialysate cultures were negative and the peritonitis responded to 10 days of treatment with intraperitoneal tobramycin and cefuroxime. Ten days after the antibiotics were stopped, however, the peritonitis relapsed and she became feverish with cloudy dialysate (white cell count $6 \times 10^{9} / 1,95 \%$ polymorphs), but she had no abdominal pain. Cultures of dialysate specimens from three consecutive 2 litre bags yielded Gram negative rods. When the results of disc sensitivity testing were known treatment was changed to intraperitoneal gentamicin $(20 \mathrm{mg}$ in alternate 2 litre exchanges) and oral co-trimoxazole (four tablets twice daily). The peritonitis then resolved, and the antimicrobial treatment was continued for a further three weeks.

Two weeks after the treatment was stopped the peritonitis recurred. No organism was isolated, and she failed to respond to further treatment with gentamicin and co-trimoxazole. The peritoneal catheter was therefore removed as the likely source of the continuing infection, and she was maintained on haemodialysis. The infection then resolved, and four weeks later a peritoneal catheter was inserted and 
continuous ambulatory peritoneal dialysis restarted. There was no further peritonitis, and successful renal transplantation was performed in January 1983.

\section{CASE 2}

A 31 year old man with chronic glomerulonephritis started continuous ambulatory peritoneal dialysis in September 1981 and had culture negative episodes of peritonitis in December 1981 and January 1982. In November 1982 he again developed peritonitis with generalised abdominal rebound tenderness, fever, and cloudy dialysate (white cell count $2.27 \times$ $\left.10^{9} / \mathrm{l}\right)$. He was treated with intraperitoneal gentamicin and oral ampicillin and cloxacillin despite nègative blood and dialysate cultures. Initially, considerable clinical improvement occurred, but during the sixth day of treatment the peritonitis relapsed. Numerous further specimens of dialysate were obtained for culture, and Gram negative rods were isolated from five separate samples. Treatment was changed to co-trimoxazole (two tablets twice daily) and intraperitoneal gentamicin $(20 \mathrm{mg}$ in alternate 2 litre exchanges). The peritonitis resolved during the next week, and treatment was continued for a further two weeks. He remained free of infection while receiving continuous ambulatory peritoneal dialysis until a successful renal transplant was performed in April 1983.

\section{CASE 3}

A 56 year old woman with adult polycystic disease started continuous ambulatory peritoneal dialysis in July 1982. Peritonitis developed in February 1983 and responded rapidly to intraperitoneal cefuroxime, although no organism was isolated on culture. She was asymptomatic until April 1983, when she developed abdominal pain, fever of $37.5^{\circ} \mathrm{C}$, and a cloudy dialysate. Gram negative rods were isolated from the dialysate, and treatment with intraperitoneal cefuroxime led to an initial resolution of fever and clearing of the dialysate. Six days later, however, peritonitis recurred accompanied by cloudy dialysate and pronounced tender erythema of the abdominal wall. Gram negative rods were again isolated from the dialysate, and intraperitoneal gentamicin $(8 \mathrm{mg}$ in each 2 litre exchange) was given with subsequent resolution of signs and symptoms. Eight days later peritonitis recurred, but it subsided within 36 hours of removal of the peritoneal dialysis catheter.

CASE 4

A 67 year old man started continuous ambulatory peritoneal dialysis in May 1980. Peritonitis due to Staphylococcus epidermidis occurred in February
1982 , and there was an episode of streptococcal peritonitis in February 1983. In August 1983 he became mildly unwell with abdominal pain, abdominal rebound tenderness, and a cloudy dialysate. Culture of the dialysate yielded Gram negative rods, and treatment was started with intraperitoneal gentamicin and vancomycin $(8 \mathrm{mg}$ and $25 \mathrm{mg}$, respectively in each 2 litre exchange). The peritonitis rapidly resolved and the dialysate cleared. Gentamicin alone was continued for 10 days, but eight days later peritonitis recurred and the same organism was again isolated from the dialysate. As there was no response to intraperitoneal vancomycin and cefuroxime the peritoneal dialysis catheter was removed, and symptoms resolved within 24 hours.

\section{CASE 5}

A 61 year old man with hypertensive nephrosclerosis began continuous ambulatory peritoneal dialysis in November 1981. He had culture negative peritonitis in November 1982 that responded to intraperitoneal cefuroxime and developed Staph epidermidis peritonitis in January 1984 that responded to intraperitoneal vancomycin. In August 1984 he presented with cloudy dialysate but had no abdominal symptoms, and he was sent home taking intraperitoneal gentamicin and vancomycin (dosages as in case 4). Culture of the dialysate yielded Gram negative rods. At his next visit the dialysate had cleared, and he remained asymptomatic.

\section{BACTERIOLOGY}

\section{Leeds (cases 1 and 2)}

Samples of dialysate were collected by the nursing staff on the ward by needle and syringe through the injection port of the drainage bag. Fluid $(10 \mathrm{ml})$ was transferred to a sterile container and a further $10 \mathrm{ml}$ inoculated into brain heart infusion and fastidiots anaerobic broths (Lab $\mathbf{M}$, Pendleton, Salford, Lancs). The samples were transported immediately to the laboratory and a white cell count performed.

A centrifuged deposit was cultured on $5 \%(\mathrm{vol} /$ vol) horse blood agar, incubated aerobically (on heated blood agar incubated under $5 \%$ carbon dioxide) and under anaerobic conditions ( $80 \%$ nitrogen, $10 \%$ hydrogen, $10 \%$ carbon dioxide). The broth cultures were incubated and subcultured after 24 and 48 hours on to solid media as above. All plates were incubated for $\mathbf{4 8}$ hours. A smear of the deposit was Gram stained.

No organisms were seen in the Gram stained smears from either patient. In both cases growth was observed on the heated blood agar plates after 48 hours and on the aerobic blood agar plate after 72 hours of incubation. All broth subcultures and repeat cultures from subsequent dialysate samples 
yielded the same isolates. Colonies were $1-2 \mathrm{~mm}$ in diameter, yellow, circular, convex, and opaque and consisted of small short Gram negative rods.

Isolates from both cases were positive for oxidase and catalase and hydrolysed aesculin slowly. API 20E identification strips (API Laboratory Products Ltd, Basingstoke, Hants) gave negative results with the exception of the oxidase reaction, and together with the results of the supplementary tests, both isolates yielded the profile number 0000004-21. This number was listed in the API analytical profile index with the comment, "good likelihood but low selectivity identification and the most likely taxa are Pseudomonas stutzeri, Achromobacter xylosoxidans, and Pseudomonas spp (other)." The isolates were subsequently referred to the Computer Identification Laboratory at the National Collection of Type Cultures (NCTC) for identification, where they were given the strain numbers CL225/82 and CL262/82, respectively.

After the second case occurred environmental samples were collected from sinks, taps, and showers on the ward. In addition, dialysis fluid and its additives - that is, antimicrobials and heparin-were sampled, as were the disinfectants used in the ward and in the patients' homes.

Samples were cultured on blood agar under $5 \%$ carbon dioxide for 72 hours and yellow colonies positive for oxidase examined further. No organisms were isolated from the dialysis fluid, additives, or disinfectants tested. Twelve isolates of yellow pigmented bacteria positive for oxidase were obtained from environmental samples, and these were tested for aesculin hydrolysis. Four isolates hydrolysed aesculin and were subsequently identified as Flavobacterium spp by API $20 \mathrm{E}$.

Oxford (cases 3, 4, and 5) For case 3 dialysate samples were collected in the same way as for the Leeds cases. On receipt in the laboratory the samples $(10 \mathrm{ml})$ were centrifuged and the deposit cultured on $5 \%(\mathrm{vol} / \mathrm{vol})$ horse blood agar incubated both aerobically and under anaerobic conditions $(80 \%$ nitrogen, $10 \%$ hydrogen, $10 \%$ carbon dioxide). In addition, an aliquot was inoculated into a Robertson's cooked meat broth. At $\mathbf{4 8}$ hours the broth was subcultured on to two blood agar plates as above. All plates were incubated for $\mathbf{4 8}$ hours.

For cases 4 and 5 dialysate was inoculated at the bedside into thioglycollate and brain heart infusion blood culture broths (Gibco Ltd, PO Box 35, Trident House, Renfrew Road, Paisley, Scotland). These were incubated for 48 hours before subculture to aerobic and anaerobic blood agar plates.

Samples from cases 3,4 , and 5 each yielded growth after 48 hours on blood agar incubated aerobically. Colonies were initially small and poorly pigmented, but after a further 24 hours of incubation they had all the characteristics of the Leeds isolates. Identification of each isolate was attempted using two systems designed for non-fermenting organisms: UNI N/F Tek (Flow Laboratories, Second Avenue Industrial Estate, Irvine, Ayrshire, Scotland) and API 20NE (API Laboratory Products Ltd, Grafton Way, Basingstoke, Hants). UNI N/F Tek identified isolates from cases 3,4 , and 5 as $P$ paucimobilis (profile numbers 406650, 406650, and 406654, respectively). API 20NE gave an identification of $P$ vesicularis for isolates from cases 3 and 4 and of $P$ paucimobilis for the last isolate (profile numbers 0460344, 0460344, and 0463745, respectively). All three isolates were referred to the NCTC for definitive identification, where they were given the reference numbers CL184/83, CL321/83, and CL360/84, respectively.

IDENTIFICATION AND SENSITIVITY TESTING

Table 1 shows the results of the biochemical tests performed on the strains at the NCTC and compares them with the characteristics of the Agrobacterium yellow group ${ }^{5}$ and of $P$ paucimobilis. ${ }^{6}$ The methods used for these tests have been described previously. ${ }^{7}$ The characteristics of the clinical strains agreed well with those of the respective reference organisms, although some of the clinical strains assigned to the Agrobacterium yellow group differed from the reference strains of that taxon in the following characters: growth at $37^{\circ} \mathrm{C}$; catalase production; gelatinase production (plate method); Tween 80 hydrolysis; and acid in ammonium salt sugar (ASS) medium from arabinose and salicin. The two taxa Agrobacterium yellow group and $P$ paucimobilis were very similar, and the most useful characters for differentiating the two were 3-ketolactose production, followed by Tween 80 hydrolysis and production of acid from ASS ethanol.

In addition to the four cases described above, the NCTC identified two further clinical isolates of Agrobacterium yellow group referred from other centres. The first isolate (CL325/82) had been cultured from the dialysate of a patient receiving continuous ambulatory peritoneal dialysis and the second (CL32/83) from fluid drained from a cranial wound. All six isolates of Agrobacterium yellow group together with the isolate of $\boldsymbol{P}$ paucimobilis were further examined in the Oxford laboratory.

Each isolate was cultured on various sensitivity testing media and at different temperatures to determine optimal conditions for sensitivity testing. All isolates grew slightly better on media containing blood. Growth at $30^{\circ} \mathrm{C}$ was in all cases equal to or slightly better than that at $37^{\circ} \mathrm{C}$. Antimicrobial sen- 
Table 1 Biochemical characteristics of Agrobacterium yellow group, of Pseudomonas paucimobilis, and of clinical strains of both taxa studied

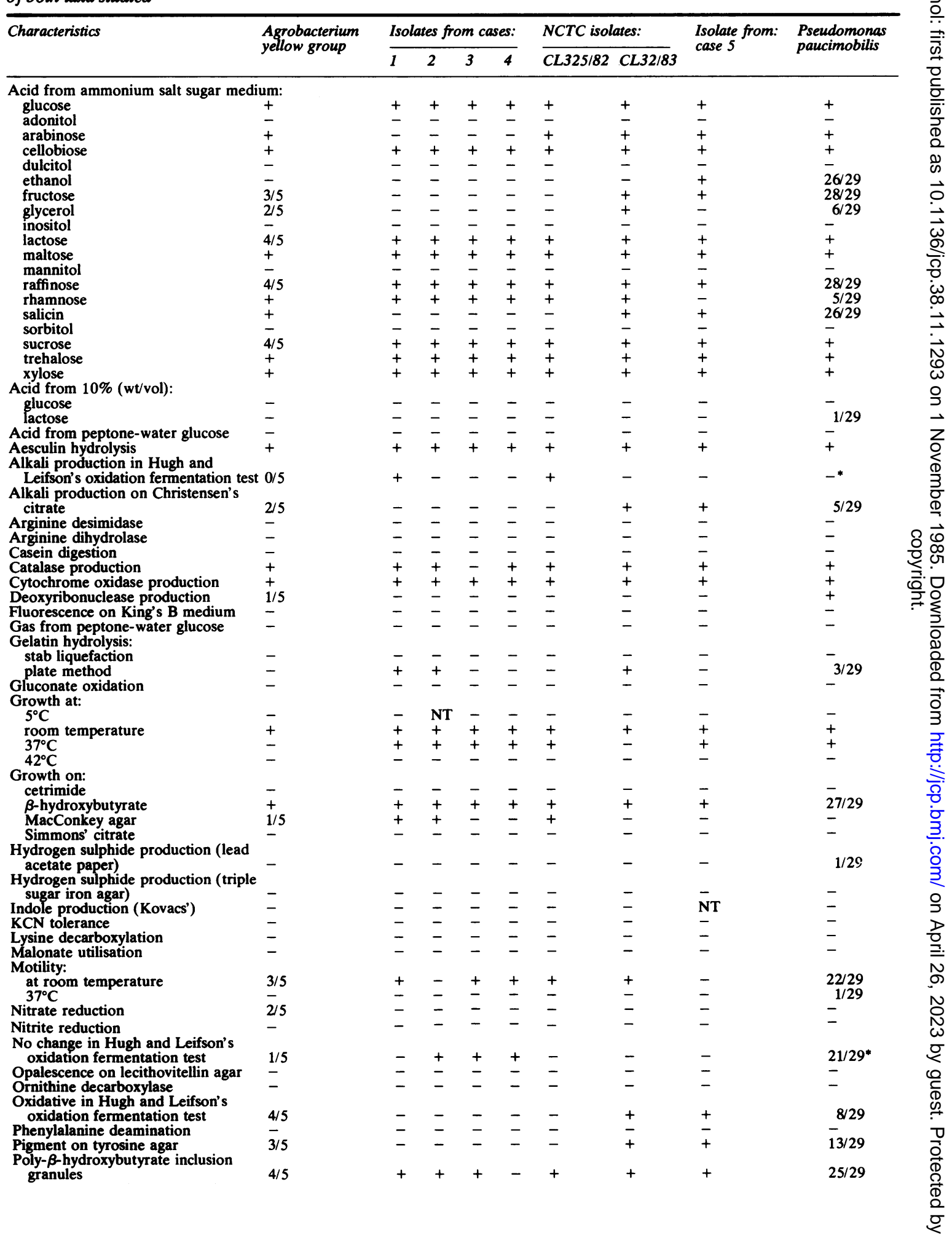


Table 1 -continued

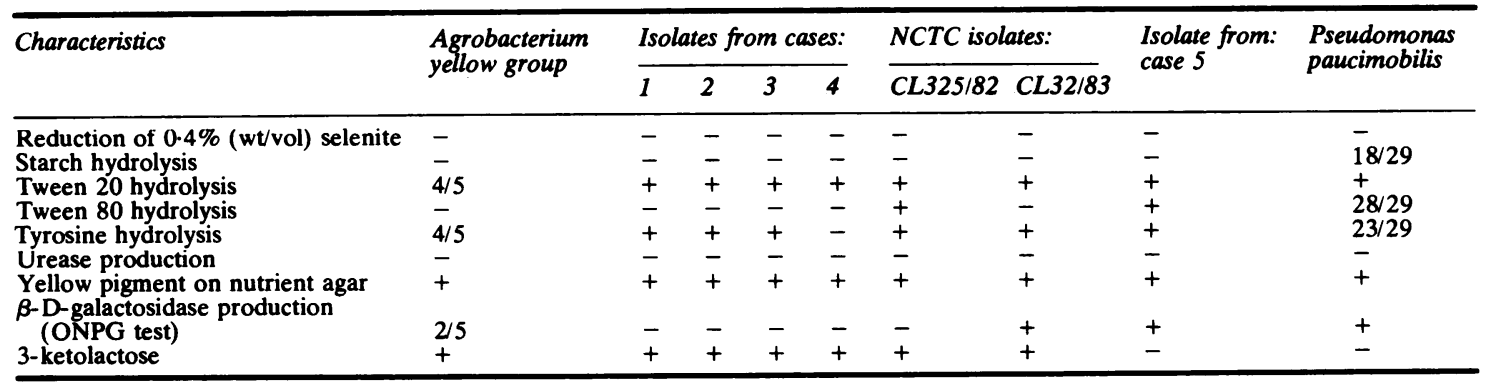

Some results for the Agrobacterium yellow group are taken from NCTC records.

$+=$ all strains tested positive; $-=$ all strains tested negative.

NT $=$ not tested.

Room temperature $=18-22^{\circ} \mathrm{C}$

*Presumed figures.

sitivities were therefore determined on Isosensitest agar with added $5 \%$ (vol/vol) lysed horse blood at $30^{\circ} \mathrm{C}$. Initially, disc diffusion sensitivity testing was performed by the comparative method. ${ }^{8}$ Plate incorporation minimum inhibitory concentrations were then determined for those antimicrobials that had given intermediate results. For both methods Escherichia coli (NCTC 10418) served as the control, and plates were incubated for 48 hours before reading. Table 2 shows the results.

Agrobacterium yellow group strains from cases 1 and 2 were further examined by Dr $T$ Preece in Leeds and were found not to produce tumours in plants.

\section{Discussion}

Agrobacteria are motile, non-sporing, obligately aerobic, Gram negative bacteria that inhabit soil, most showing little growth above $29^{\circ} \mathrm{C}$. They are the only organisms that characteristically produce 3-ketolactose, and certain species are important plant pathogens, initiating cell proliferation that results in gall formation. Several authors ${ }^{4}$ have expressed their disagreement with the classification of the organism. ${ }^{3}$ A classification based on biochemical criteria has been proposed. ${ }^{5}$ This describes four taxa, the fourth being a previously unrecognised group that does not produce tumours when inoculated into plants. The five strains assigned to this group were NCTC strains 10590 and 10591 and NCPPB (National Collection of Plant Pathogenic Bacteria, Hatching Green, Harpenden, Herts) strains 2661,2662 , and 2663 . The group has been tentatively named Agrobacterium yellow group because the strains produce 3-ketolactose and are yellow pigmented. Although rRNA studies show that strains NCTC 10590 and 10591 belong in the fourth rRNA superfamily, however, as do the true agrobacteria, the rRNA cistrons of these two strains are more closely related to the rRNA cistrons of the type strain of Flavobacterium capsulatum than to those of Agrobacterium. ${ }^{4}$ These rRNA studies showed that the closest relatives to $F$ capsulatum

Table 2 Disc diffusion sensitivities and minimum inhibitory concentrations $(\mu \mathrm{g} / \mathrm{ml})$ of Agrobacterium yellow group and $P$ paucimobilis clinical strains

\begin{tabular}{|c|c|c|c|c|c|c|c|}
\hline \multirow[t]{2}{*}{ Antimicrobial agent } & \multicolumn{4}{|c|}{ Isolates from cases: } & \multicolumn{2}{|c|}{ NCTC isolates: } & \multirow{2}{*}{$\begin{array}{l}\text { Isolate } \\
\text { from case } 5\end{array}$} \\
\hline & 1 & 2 & 3 & 4 & $C L 325 / 82$ & CL32/83 & \\
\hline $\begin{array}{l}\text { Cephalothin } \\
\text { Cefuroxime } \\
\text { Cefotaxime } \\
\text { Ceftazidime } \\
\text { Ticarcillin } \\
\text { Piperacillin } \\
\text { Netilmicin } \\
\text { Amikacin } \\
\text { Chloramphenicol } \\
\text { Sulphamethoxazole } \\
\text { Trimethoprim } \\
\text { Ampicillin } \\
\text { Gentamicin }\end{array}$ & $\begin{array}{l}\mathbf{R} \\
\mathbf{R} \\
\mathbf{R} \\
\mathbf{R} \\
\mathbf{S} \\
\mathbf{R} \\
\mathbf{R} \\
\mathbf{R} \\
\mathbf{R} \\
<2 \\
4 \\
32 \\
4\end{array}$ & $\begin{array}{l}\mathbf{R} \\
\mathbf{R} \\
\mathbf{R} \\
\mathbf{R} \\
\mathbf{S} \\
\mathbf{R} \\
\mathbf{R} \\
\mathbf{R} \\
\mathbf{R} \\
<2 \\
4 \\
32 \\
4\end{array}$ & $\begin{array}{r}\mathbf{R} \\
\mathbf{R} \\
\mathbf{R} \\
\mathbf{R} \\
\mathbf{S} \\
\mathbf{R} \\
\mathbf{R} \\
\mathbf{R} \\
\mathbf{R} \\
<2 \\
4 \\
32 \\
4\end{array}$ & $\begin{array}{r}\mathbf{R} \\
\mathbf{R} \\
\mathbf{R} \\
\mathbf{R} \\
\mathbf{S} \\
\mathbf{R} \\
\mathbf{R} \\
\mathbf{R} \\
\mathbf{R} \\
<2 \\
4 \\
32 \\
4\end{array}$ & $\begin{array}{l}\mathbf{R} \\
\mathbf{R} \\
\mathbf{R} \\
\mathbf{R} \\
\mathbf{S} \\
\mathbf{R} \\
\mathbf{R} \\
\mathbf{R} \\
\mathbf{R} \\
<2 \\
4 \\
32 \\
4\end{array}$ & $\begin{array}{l}\mathbf{R} \\
\mathbf{R} \\
\mathbf{R} \\
\mathbf{R} \\
\mathbf{S} \\
\mathbf{R} \\
\mathbf{S} \\
\mathbf{S} \\
\mathbf{S} \\
\quad 64 \\
>16 \\
32 \\
\quad 0.5\end{array}$ & $\begin{array}{l}\text { R } \\
\text { S } \\
\text { S } \\
\mathbf{R} \\
\text { S } \\
\mathbf{R} \\
\mathbf{S} \\
\mathbf{S} \\
\mathrm{S} \\
\mathrm{NT} \\
>16 \\
\quad 1 \\
\quad 0.5\end{array}$ \\
\hline
\end{tabular}

$R=$ resistant,$S=$ sensitive, $N T=$ not determined. 
(itself misplaced in Flavobacterium) are a few yellow pigmented organisms including, apart from NCTC strains 10590 and $10591, F$ devorans, $P$ azotocolligans, and $P$ paucimobilis. ${ }^{\prime}$ It should be appreciated that the Agrobacterium yellow group as originally described comprised only five strains from plants that were heterogeneous in their biochemical characteristics. The clinical strains from continuous ambulatory peritoneal dialysis fluid assigned to the group differ in some characters from the reference strains, but this may be due to their differences in origin, because the group was described from so few strains or because the clinical strains belong in a different (but related) taxon from the reference strains. The group obviously requires further taxonomic study.

True members of the genus Agrobacterium have only occasionally been isolated from clinical specimens. The Center for Disease Control, Atlanta, United States, received 37 isolates over 12 years, ${ }^{10}$ and further isolates have been reported by Lautrop" and Kiredjian. ${ }^{12}$ In these reports the agrobacteria were usually isolated in association with other bacteria and therefore thought to represent contaminants. In only one case - that of prosthetic valve endocarditis-was an Agrobacterium sp considered to have been the infecting organism. ${ }^{13}$

$\boldsymbol{P}$ paucimobilis has also rarely been reported as a pathogen in man, ${ }^{14-17}$ although it is well recognised as an organism associated with the hospital environment, ${ }^{6}$ where it may cause outbreaks of infection. ${ }^{18}$ A recent report describes two cases of peritonitis in patients receiving peritoneal dialysis caused by this organism. ${ }^{19}$

In case 5 the isolate was initially considered to be Agrobacterium yellow group because of its cultural similarity to the previous isolates (cases 3 and 4). Furthermore, the UNI N/F Tek system gave the same species identification as for the isolates from cases 3 and 4 . The API 20NE, however, indicated a different species for the isolate from case 5, and the antimicrobial susceptibility profile was also quite different from that of the previous isolates (Table 2). The API 20NE system lists the species Agrobacterium radiobacter (synonym: $A$ tumefaciens ${ }^{5}$ ) in the database, but, clearly, neither commerical system can distinguish Agrobacterium yellow group from related taxa.

Optimal antimicrobial treatment remains to be determined as only one of the four cases of peritonitis due to Agrobacterium yellow group responded to chemotherapy (case 2). The remaining three cases required removal of the catheter for complete resolution. Ticarcillin and sulphamethoxazole were the only antimicrobials to which all four strains were sensitive. The case of peritonitis due to
$P$ paucimobilis responded well to intraperitoneal gentamicin. In the previous report of peritonitis caused by this organism one of the two cases responded to intraperitoneal co-trimoxazole, whereas the second required removal of the catheter. ${ }^{14}$

Notably, we saw four cases of Agrobacterium yellow group peritonitis in two continuous ambulatory peritoneal dialysis centres within two years. Considering the time interval between individual cases, cross infection within units would seem to be unlikely. An environmental source for the infections at Leeds was sought but agrobacteria were not recovered, and there was no evidence to implicate contaminated dialysis fluid as the source of infection. A further interesting feature is that all five continuous ambulatory peritoneal dialysis isolates of Agrobacterium yellow group examined (including CL325/ 82) shared the same antimicrobial sensitivity profile and generally the same pattern of biochemical characteristics, which were distinct from those of other non-dialysate isolates of this taxon examined (CL32/83; Tables 1 and 2, and unpublished data).

In conclusion, we report these five cases of peritonitis in the hope that any future yellow pigmented isolates from patients receiving continuous ambulatory peritoneal dialysis are fully characterised, so that the source of these infections may be further elucidated. Currently available commercial systems are apparently inadequate for the identification of this group of organisms.

We thank Dr DO Oliver of the renal unit, Churchill Hospital, Oxford, and Dr AM Brownjohn of the renal unit, Leeds General Infirmary, for permission to report these cases; Dr TF Preece of the department of plant sciences, University of Leeds, for his phytopathogenic studies; and PS Humphry for technical help.

\section{References}

' Anonymous. Ambulatory peritonitis [Editorial]. Lancet 1982; i: 1104-5.

${ }^{2}$ Ramos JM, Gokal R, Siamopolous K, Ward MK, Wilkinson R, Kerr DNS. Continuous ambulatory peritoneal dialysis: three years experience. $Q J$ Med 1983;52:165-86.

${ }^{3}$ Allen ON, Holding AJ. Genus II. Agrobacterium Conn 1942, 359 Nom gen cons. Opin 33, Jud Comm 1970, 10. In: Buchanan RE, Gibbons NE, eds. Bergey's manual of determinative bacteriology. 8th ed. Baltimore: Williams and Wilkins, 1974:264.

4 Kersters K, De Ley J. Genus III. Agrobacterium Conn 1942, 359. In: Krieg NR, ed. Bergey's manual of systematic bacteriology. Vol. 1. Baltimore: Williams and Wilkins 1984:244.

s Holmes B, Roberts P. The classification, identification and nomenclature of agrobacteria. J Appl Bacteriol 1981;50: 443-67. 
' Holmes B, Owen RJ, Evans A, Malnick H, Willcox WR. Pseudomonas paucimobilis, a new species isolated from human clinical specimens, the hospital environment, and other sources. Int J Syst Bacteriol 1977;27:133-46.

7 Holmes B, Lapage SP, Malnick H. Strains of Pseudomonas putrefaciens from clinical material. J Clin Pathol 1975;28:149 55.

${ }^{8}$ Waterworth PM. Laboratory methods. In: Garrod LP, Lambert HP, O'Grady F. Antibiotic and chemotherapy. 5th ed. Edinburgh: Churchill Livingstone, 1981.

' Bauwens $M$, De Ley J. Improvements in the taxonomy of Flavobacterium by DNA:rRNA hybridizations. In: Reichenbach H, Weeks OB, eds. The Flavobacterium-Cytophaga group. Weinheim: Verlag Chemie, 1981:27.

${ }^{10}$ Riley PS, Weaver RE. Comparison of thirty-seven strains of Vd-3 bacteria with Agrobacterium radiobacter: morphological and physiological observations. J Clin Microbiol 1977;5:1727.

"Lautrop H. Agrobacterium spp isolated from clinical specimens. Acta Pathol Microbiol Scand 1967;187 (suppl): 63-4.

${ }^{12}$ Kiredjian M. Le genre Agrobacterium peut-il être pathogène pour l'homme? Médicine et Maladies Infectieuses 1979;9:233-5.

${ }^{13}$ Plotkin GR. Agrobacterium radiobacter prosthetic valve endocarditis. Ann Intern Med 1980;93:839-40.

14 Peel MM, Davis JM, Armstrong WLH, Wilson JR, Holmes B. Pseudomonas paucimobilis from a leg ulcer on a Japanese seaman. J Clin Microbiol 1979;9:561-4.

is Hajiroussou V, Holmes B, Bullas J, Pinning CA. Meningitis caused by Pseudomonas paucimobilis. J Clin Pathol 1979;32: 953-5.

16 Slotnick IJ, Hall J, Sacks H. Septicemia caused by Pseudomonas paucimobilis. Am J Clin Pathol 1979;72:882-4.

17 Southern PM, Kutscher AE. Pseudomonas paucimobilis bacteremia. J Clin Microbiol 1981;13:1070-3.

${ }^{18}$ Crane LR, Tagle LC, Palutke WA. Outbreak of Pseudomonas paucimobilis in an intensive care facility. J Am Med Assoc 1981;246: 985-7.

${ }^{14}$ Glupczynski Y, Hansen W, Dratwa M, et al. Pseudomonas paucimobilis peritonitis in patients treated by peritoneal dialysis. J Clin Microbiol 1984;20: 1225-6.

Requests for reprints to: Dr RA Swann, Department of Microbiology and Public Health Laboratory, Level 5, Phase III, Leicester Royal Infirmary, Infirmary Square, Leicester LE1 5WW, England. 